\title{
ORE MINERALS FROM KUROKO TYPE DEPOSIT OF TOYA-TAKARADA MINE, HOKKAIDO, JAPAN
}

\section{MINERAL BIJIH DARI ENDAPAN TIPE KUROKO PADA TAMBANG TOYA-TAKARADA, HOKKAIDO, JEPANG}

\author{
Euis Tintin Yuningsih \\ Faculty of Geology, Padjadjaran University, Bandung-Indonesia \\ etintiny@yahoo.com
}

\begin{abstract}
Toya-Takarada mine is Au- and Ag-rich Kuroko-type deposit located in Takarada, Toya-mura, southwest Hokkaido, Japan. The deposits were hosted in rhyolitic tuff and mudstone of Middle Miocene age. Ore samples of fine-grained black ore, vuggy black-yellow ore, granular vuggy black ore, quartz-sulfide ore and massive quartz-barite ore were studied to identify the ore minerals association in the Toya-Takarada mine. The ore minerals are dominated by sphalerite, galena, chalcopyrite and pyrite with fewer amounts of electrum, tetrahedrite-tennantite, and other sulfosalt minerals with secondary mineral of covellite.
\end{abstract}

The quantitative chemical analysis of ore minerals by EPMA indicated that FeS contents in sphalerite is low (0.3-1.2 mol.\%) in all kinds of ore samples. Small grains of electrum as inclusions in pyrite are identified in vuggy black-yellow ore with Ag content around 32-33 atm \%.

In general, the silver minerals in Kuroko-type deposits occurred mainly in the black and yellow ores zone dominantly composed of sphalerite, galena, pyrite, chalcopyrite and barite as a form of electrum and/or argentian tetrahedrite-tennantite series. Thus, the massive quartz-barite ore sample of Toya-Takarada mine are also contain some rare silver sulfosalt minerals such as proustite, Cu-rich pearceite, geocronite-jordanite and fizelyite. Those minerals were found together in association with sphalerite. It seems that sphalerite was crystallized first followed by proustite and $\mathrm{Cu}$-rich pearceite, then geocronite-jordanite and fizelyite are crystallized later.

Sphalerites from quartz-sulfide ore of Toya-Takarada contain some fluid inclusions and measured homogenization temperatures are in the range of $164-247^{\circ} \mathrm{C}$ (av. $208^{\circ} \mathrm{C}$ ) with salinity ranging from 1.9 to $4.7 \mathrm{wt} . \% \mathrm{NaCl}_{\text {equiv. }}$ (av. $3.9 \mathrm{wt} \% \mathrm{NaCl}_{\text {equiv. }}$. The mineral assemblage, iron content in sphalerite and silver content in electrum were indicated that sulfur fugacity was slightly higher during ore mineralization in Toya-Takarada mine.

Keywords: black ore, fizelyite, geocronite-jordanite, sphalerite, sulfur fugacity, vuggy blackyellow ore

\section{ABSTRAK}

Toya-Takarada merupakan tipe cebakan Kuroko kaya emas dan perak yang berlokasi di Takarada, Toya-mura, sebelah tenggara Hokkaido, Jepang. Endapan Toya-Takarada memiliki hostrock tuf riolitik dan batulempung berumur Miosen Tengah. Conto bijih yang diambil dari fine-grained black ore, vuggy black-yellow ore, granular vuggy black ore, quartz-sulfide ore and massive quartz-barite ore dipelajari untuk mengetahui asosiasi mineral bijih yang terdapat di cebakan Toya-Takarada. Mineral bijih didominasi oleh sfalerit, galena, kalkopirit, dan pirit dengan sedikit elektrum, tetrahedrit-tenantit, dan mineral sulfosalt lainnya dengan kehadiran mineral sekunder kovelit. 
Analisis kimia kuantitatif dari mineral bijih dengan EPMA menunjukkan kandungan FeS yang rendah (0.3 s.d 1.2 mol.\%) dalam sfalerit di seluruh tipe conto bijih. Butiran berukuran kecil dari elektrum hadir sebagai inklusi dalam pirit terdapat pada vuggy black-yellow ore dengan kandungan Ag sekitar 32 s.d 33 atm. \%.

Secara umum mineral yang mengandung perak dalam cebakan tipe kuroko terdapat dalam zona black dan yellow ores yang didominasi oleh sfalerit, galena, pirit, kalkopirit dan barit dalam bentuk elektrum, dan/atau seri Ag-tetrahedrit-tenantit. Namun conto dari massive quartz-barite ore yang diambil dari Toya-Takarada juga mengandung mineral Ag-sulfosalt jarang seperti prostit, persit kaya $\mathrm{Cu}$, geokronit-jordanit dan fizelit. Mineral-mineral tersebut ditemukan bersama-sama berasosiasi dengan sfalerit. Terlihat bahwa sfalerit terbentuk pertama dan diikuti oleh prostit dan persit kaya $\mathrm{Cu}$, sementara geokronit-jordanit dan fizelit terbentuk kemudian.

Sfalerit dari quartz-sulfide ore endapan Toya-Takarada mengandung inklusi fluida dan mengindikasikan temperatur homogenitas dalam kisaran 164 s.d $247^{\circ} \mathrm{C}$ (rata-rata $208^{\circ} \mathrm{C}$ ) dengan salinitas berkisar dari 1.9 sampai 4.7 wt.\% NaClequiv. (rata-rata 3.9 wt.\% NaClequiv.). Kelompok mineral bijih, kandungan besi dalam sfalerit dan kandungan perak dalam elektrum mengindikasikan kondisi fugasitas sulfur yang tinggi pada saat pembentukan mineral di cebakan Toya-Takarada.

Kata kunci: black ore, fizelit, geokronit-jordanit, sfalerit, fugasitas sulfur, vuggy black-yellow ore

\section{INTRODUCTION}

The Kuroko deposits in Japan are formed during a very limited period, around $13 \mathrm{Ma}$ and it is in association with late Oligocene or early Miocene of mafic to felsic volcanic cycle late-stage felsic rocks. The deposits are predominantly massive, stratiform sulphide ores with associated underlying stockwork ores of Miocene age in the Green Tuff volcanic sequences and occur in narrow zones parallel to island arcs. The Benioff zone is at about $150-\mathrm{km}$ depth below the Kuroko zones, which were areas of high heat flow during the Miocene (Sato, 1977).

Kuroko-type deposits are named after the type locality in the Miocene Green Tuff belt of Japan and the setting is thought to be a submarine rifted basin within an oceanic volcanic arc. The Kuroko deposits are polymetallic, yielding copper, lead, zinc, and often gold as an important by-product with principal ore minerals are chalcopyrite, galena and sphalerite. Chalocopyrite-rich (pyrite-chalcopyrite-quartz) ore is known as 'yellow ore' and galena- and sphalerite-rich (galena-sphalerite-barite) ore is known as 'black ore' and there is a zonation of ore types.

The host rocks are mainly acid volcanic rocks, and a rhyolite dome is a common feature. Previous studies have shown temperatures of $200-350^{\circ} \mathrm{C}$ for ore mineralization. They are larger than the Cyprus-type massive sulphide deposits, but rarely exceed $100 \mathrm{Mt}$ of ore. However, mining districts can contain large numbers of deposits (the green tuff belt has over 100 deposits over $800 \mathrm{~km}$ strike length).

The purpose of present study is to identify ore minerals assemblage from some ores body of Kuroko-type deposit of ToyaTakarada, Southwestern Hokkaido, Japan. The physico-chemical condition of ore formation is also reconstructed together with temperature and ore mineral composition study.

\section{Regional Geology}

The geology of southwestern Hokkaido is characterized by intensive volcanisms of Miocene to Plio-Pleistocene age. The studied area, eastern margin of Lake Toya, 
is one of the centers of the volcanic field where Miocene andesitic and dacitic lavas and pyroclastic rocks (Osarugawa Formation), Pliocene dacitic tuff (Kitayuzawa Formation) and plagio-ryolite and Plio-Pleistocene andesite lavas are distributed. The volcanisms subsequently caused a concealed alteration zone, and mineralization (Marumo, 1985).

Kuroko and Kuroko-type deposits are distributed in the Shakotan-Toya district, southwest Hokkaido. The mode of occurrence of Kuroko-type deposits is somewhat different from the typical kuroko deposits in the Hokuroku district. For example, the Kuroko-type deposits lack any bedded ore-body (Sawai and Itaya, 1993).

Toya-Takarada mine is Au- and Ag-rich Kuroko-type deposit located in Takarada, Toya-mura, southwestern Hokkaido, Japan (Figures 1A \& 1B). Toya - Takarada mine is occurred as Kuroko massive sulfide deposit in ryolitic-dacitic tuff breccia in the upper part of the Middle Miocene Osarugawa Formation (Marumo, 1985; Figure 2). The Osarugawa Formation is assumed to be continental to marine sediments, and overlies unconformably Mesozoic sediments and Paleogene granitic rocks. Marumo and Sawai (1986) indicated that samples from the Horobetsu and Kagenosawa Au-Ag-Cu-Pb-Zn disseminated and vein-type deposits together with the Toya Takarada and Minamishiraoi kuroko-type deposits give K$\mathrm{Ar}$ age values generally in the range of 12.3 to $14.2 \mathrm{Ma}$ indicating that the ages of the mineralizations of these deposits were similar to those of the kuroko deposits in Hokuroku area .

Tuff breccia, the host of the deposit, has been subjected to smectite alteration. The deposit was mined in 1934 to 1942 and in 1951 to 1952 with the largest ore body approximately $50 \mathrm{~m} \times 18 \mathrm{~m} \times 30 \mathrm{~m}$. There are many gypsum deposits associated with mudstone beds (Figure 1C). Kaolinite in the alteration zone was also mined.

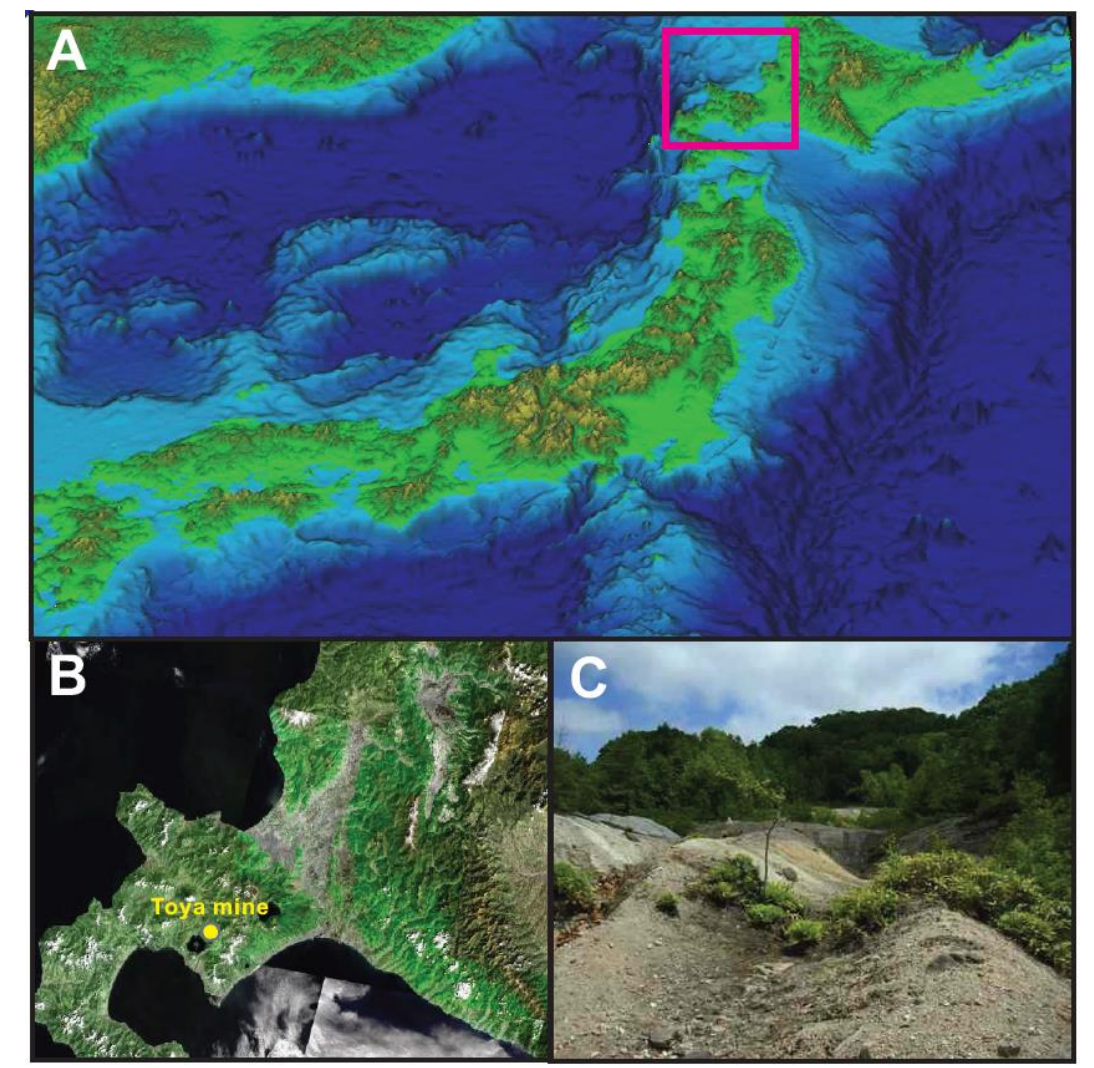

Figure 1. Map of Japan (A), location of the Toya-Takarada mine in Southwest Hokkaido (B) and the damp photograph of Toya-Takarada mine (C). 


\section{MAKALAH ILMIAH}

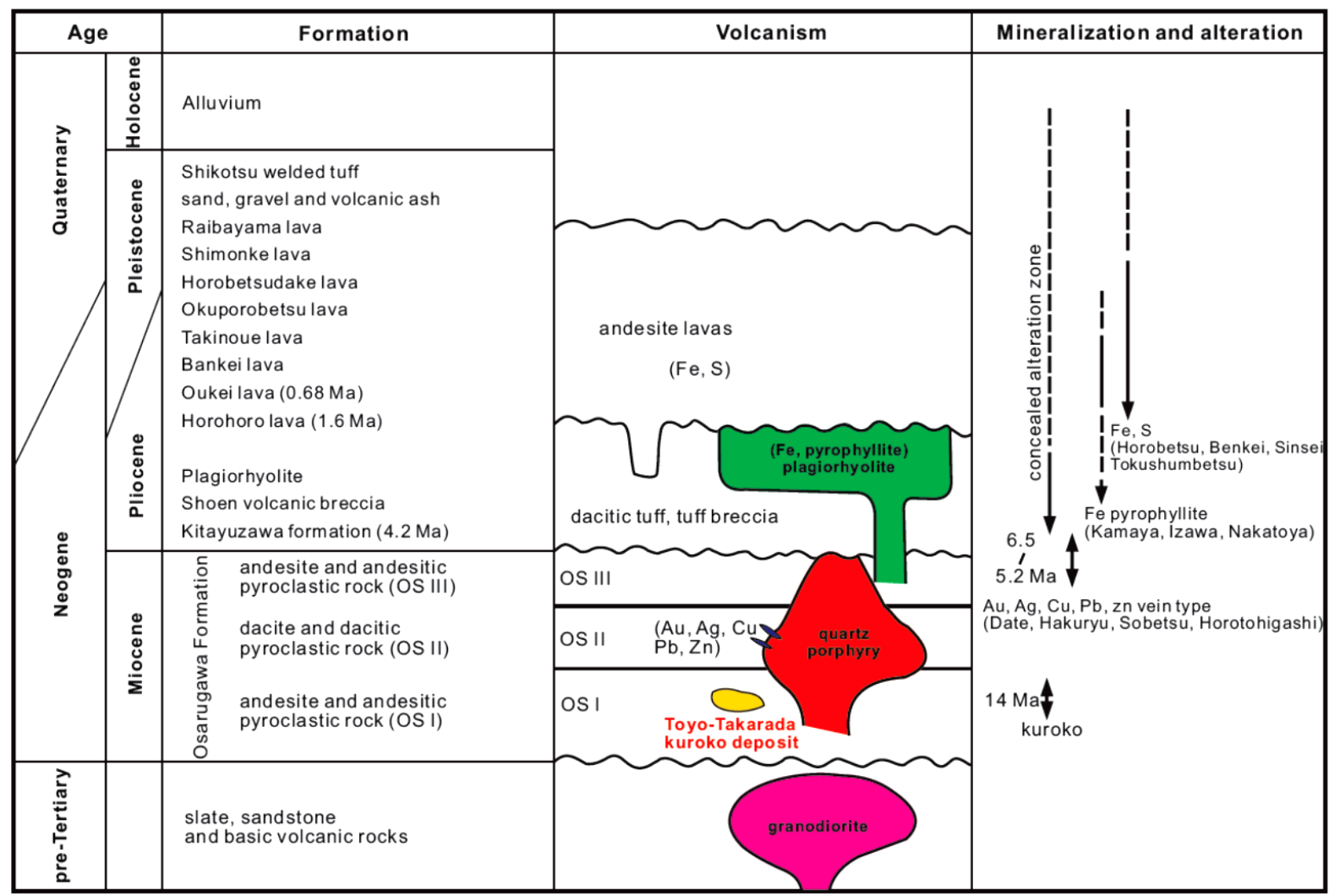

Figure 2. Summary of stratigraphic sequence, volcanism, mineralization and alteration of Toya, Southwestern Hokkaido (modified after Marumo, 1985).

\section{METHODOLOGY}

The determination of mineralogy with the chemistry and mode occurrences of ore minerals from the Toya - Takarada mine is based on the study of samples from the Hokkaido University Museum collection. Megascopic investigation hand specimens and microscopic of the selected doubly thin sections and polished sections have been used for the purpose of study.

The composition of the ore minerals were measured with electron probe mineral analyzer (EPMA) using JEOL 733 electron microprobe at the Hokkaido University. Standards using natural chalcopyrite, InP, $\mathrm{MnS}, \mathrm{CdS}, \mathrm{FeAsS}, \mathrm{Sb}_{2} \mathrm{~S}_{3}, \mathrm{PbS}, \mathrm{SnS}, \mathrm{HgS}$, $\mathrm{ZnS}$ and elemental Se, Au, Ag, Te. The probe was operating at $20 \mathrm{kV}$ voltage and the beam currents of $10 \mathrm{nA}$ was focused to 1-10 $\mu \mathrm{m}$ diameters with peak counting times was $20 \mathrm{~s}$. The X-ray lines measured were As, Se, Te, Cd, Ag, Bi and $\mathrm{Sb}(\mathrm{L} \alpha), \mathrm{S}$, $\mathrm{Cu}, \mathrm{Zn}, \mathrm{Fe}$ and $\mathrm{Mn}(\mathrm{Ka})$ and $\mathrm{Pb}, \mathrm{Au}$ and $\mathrm{Hg}$ $(\mathrm{Ma})$. The raw data was corrected by ZAF correction. The X-ray energy-dispersive and backscatter electron image with JEOL JSM-5310 scanning electron microscope were also used to mapping the coexistence ore minerals. Doubly polished thin sections of quartz and sphalerite samples were prepared on $200 \mu \mathrm{m}$ thickness for the fluid inclusion measurements. Microthermometric measurement was performed on a Linkam THMSG 600 system attached to a Nikon transmitted-light microscope at Hokkaido University.

\section{RESULTS AND DISCUSSIONS}

\section{Ore Mineralogy}

Ore samples of fine-grained black ore, vuggy black-yellow ore, granular vuggy black ore, quartz-sulfide ore and massive quartz-barite ore were studied to identify the ore minerals association in the ToyaTakarada mine. The fine-grained black ore is dominated by pyrite, sphlerite, galena, 
chalcopyrite and tetrahedrite. Vuggy blackyellow ore composed of abundant sphalerite, pyrite, chalcopyrite and followed with tetrahedrite. Granular vuggy black ore contains trace of electrum and tetrahedritetennantite, with dominant of sphalerite, pyrite, galena and chalcopyrite. Quartzsulfide ore also contains base metal minerals of sphalerite, pyrite, galena and chalcopyrite with tetrahedrite occurrence. The secondary covellite mineral occurs in fine-grained black ore, vuggy black-yellow ore and granular vuggy black ore (Figure 3 ). Paragenetic sequence of ore minerals in each ore type are presented in Figure 4.

The massive quartz-barite ore sample of Toya-Takarada mine is interesting with the occurrence of some rare silver sulfosalts minerals of cupro-pearceite, jordanite and fizelyite (Yuningsih and Matsueda, 2013). Those minerals were found together in association with sphalerite (Figure 5). It seems that sphalerite was crystallized first followed by proustite and Cu-rich pearceite, then geocronite-jordanite and fizelyite are crystallized later. Fizelyite has dark gray to gray in color with metallic luster, and shows distinct anisotropism with creamy white color under the reflective microscope. Geocronite-jordanite has gray to pale gray in color and microscopically shows pure white color with weak pleochroism and moderate anisotropism. forms a series with jordanite.

Figure 6 illustrate the mineralization sequence of sulfosalt minerals at the ToyaTakarada mine observed under thin section investigation. It seems that sphalerite was crystallized first followed by proustite and Cu-rich pearceite, then geocronitejordanite and fizelyite are crystallized later. In addition to above silver sulfosalt mineralization in Toya-Takarada mine, Shimizu et al. (1999) also identified samples containing TI-bearing minerals occur as breccias in the Kuroko massive sulfide ores, which pointed that these $\mathrm{TI}$ mineralization were preceded the Kuroko mineralization. Three TI minerals identified were hutchinsonite, chabournéite and unnamed $\left[(\mathrm{TI}, \mathrm{Ag})_{2} \mathrm{~Pb}_{6}(\mathrm{As}, \mathrm{Sb})_{16} \mathrm{~S}_{31}\right]$ with two associated minerals such as getchellite and $\mathrm{Ag}$-bearing luzonite.

\section{Chemical Composition of Ore Mineral}

The ore minerals composition was identified with microprobe (Electron-Probe Mineral Analyzer/EPMA). Mostly of common sulfide minerals such as sphalerite, chalcopyrite, pyrite and galena has the ideal International Mineral Association/IMA-approved formula. The quantitative chemical analyses of ore minerals by EPMA indicated that sphalerite with calculated formula $\mathrm{Zn}_{1.0} \mathrm{~S}_{1.0}$ has low FeS contents (0.3-1.2 mol.\%) in all kinds of ore samples. Other sulfides ore chalcopyrite with calculated formula $\mathrm{Cu}_{1.2} \mathrm{Fe}_{0.8} \mathrm{~S}_{2.0} ;$ pyrite $\mathrm{Fe}_{1.0} \mathrm{~S}_{2.0} ;$ galena $\mathrm{Pb}_{1.0} \mathrm{~S}_{1.0} ;$ and covellite $\mathrm{Cu}_{1.0} \mathrm{~S}_{1.0}$. Small grains of electrum as inclusions in pyrite are identified in vuggy black-yellow ore with $\mathrm{Ag}$ content around 32-33 atm.\% and calculated formula $\mathrm{Au}_{1.3} \mathrm{Ag}_{0.7}$. . The low $\mathrm{Ag}$ content in electrum is also reported from other siliceous and yellow ores of Kurokotype deposits in Japan, such as of Nurukawa deposit contains about 20 atm.\% of silver. In contrast, the black ore usually contain higher Ag in electrum (Shikazono \& Shimizu, 1988).

The calculated formulas of silver sulfosalt minerals (Table 1) in average are $\left.\mathrm{Cu}_{10.3} \mathrm{Fe}_{0.4} \mathrm{Ag}_{0.1} \mathrm{Zn}_{1.3}\right)_{12.1}\left(\mathrm{Sb}_{1.5} \mathrm{As}_{2.1}\right)_{3.6} \mathrm{~S}_{13.2}$ for tetrahedrite-tennantite; $\mathrm{Ag}_{3.3}\left(\mathrm{As}_{0.7} \mathrm{Cu}_{0.1}\right)_{0.8} \mathrm{~S}_{3.0}$ for proustite (7 apfu/N=3); $\mathrm{Ag}_{3.7} \mathrm{Cu}_{7.4}\left(\mathrm{As}_{3.4} \mathrm{Sb}_{0.2}\right)_{3.6} \mathrm{~S}_{14.3}$ for Cu-rich pearceite (29 apfu/ $\mathrm{N}=6)$; $\left(\mathrm{Pb}_{14.1} \mathrm{Ag}_{0.1}\right)_{14.2}\left(\mathrm{As}_{1.4} \mathrm{Sb}_{4.2}\right)_{5.6} \mathrm{~S}_{23.1} \quad$ for geocronite-jordanite (43 apfu/ $\mathrm{N}=4)$; and $\mathrm{Ag}_{4.2} \mathrm{~Pb}_{12.7}\left(\mathrm{Sb}_{19.9} \mathrm{As}_{1.1}\right)_{21.0} \mathrm{~S}_{50.1}$ for fizelyite (88 apfu/N=9). 


\section{MAKALAH ILMIAH}
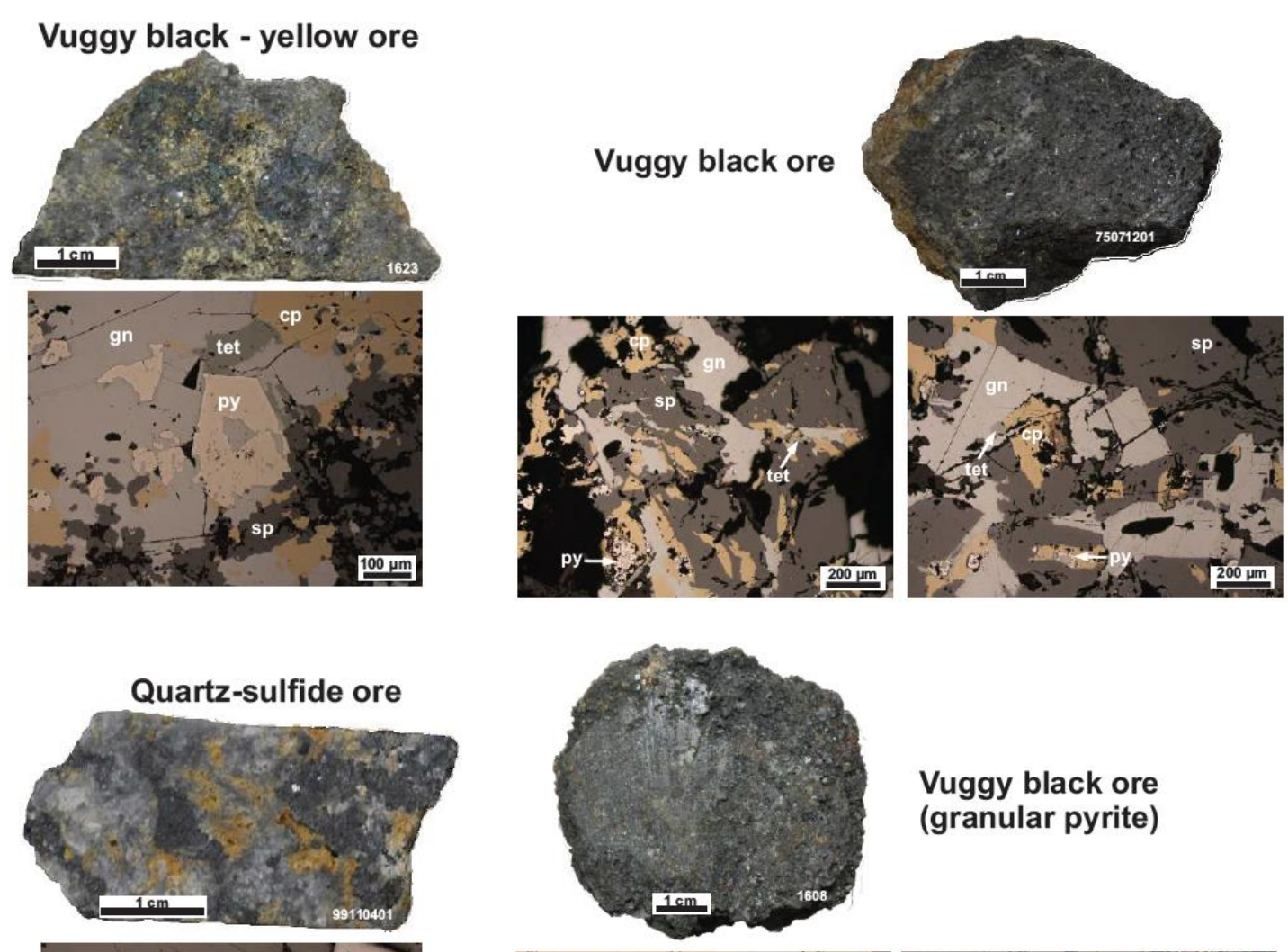

\section{Vuggy black ore (granular pyrite)}
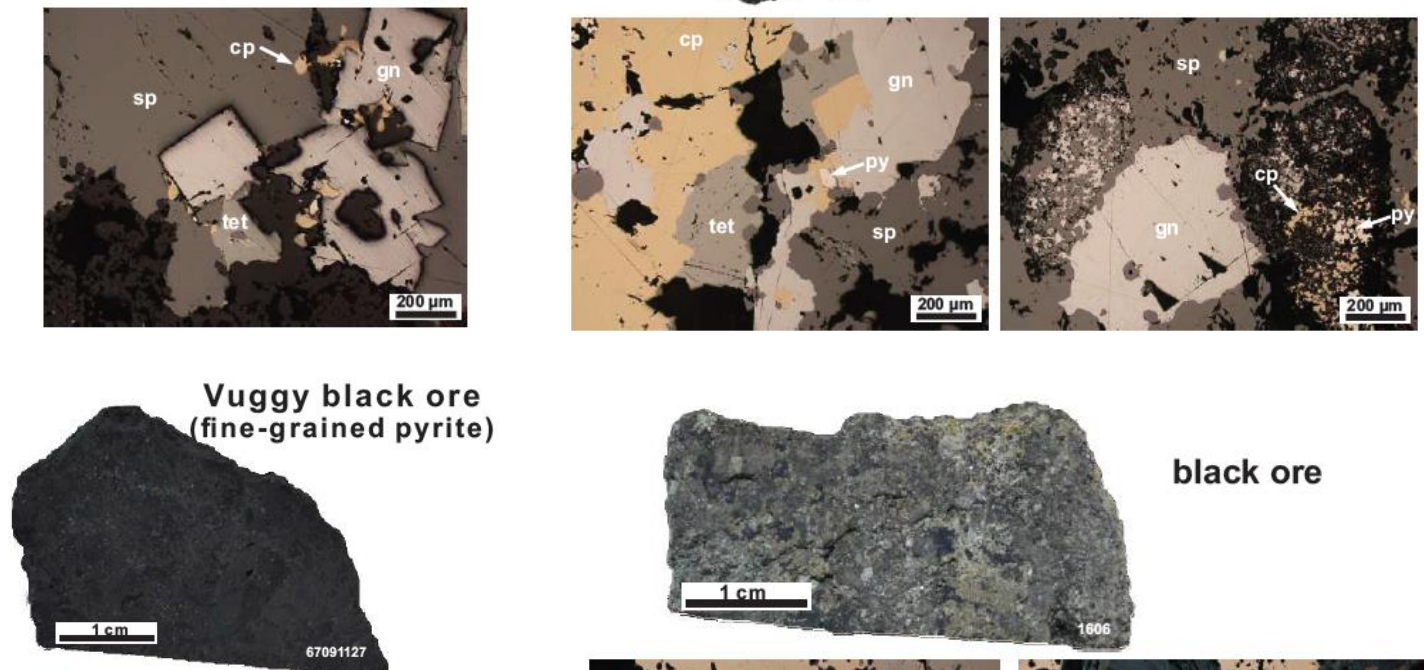

black ore
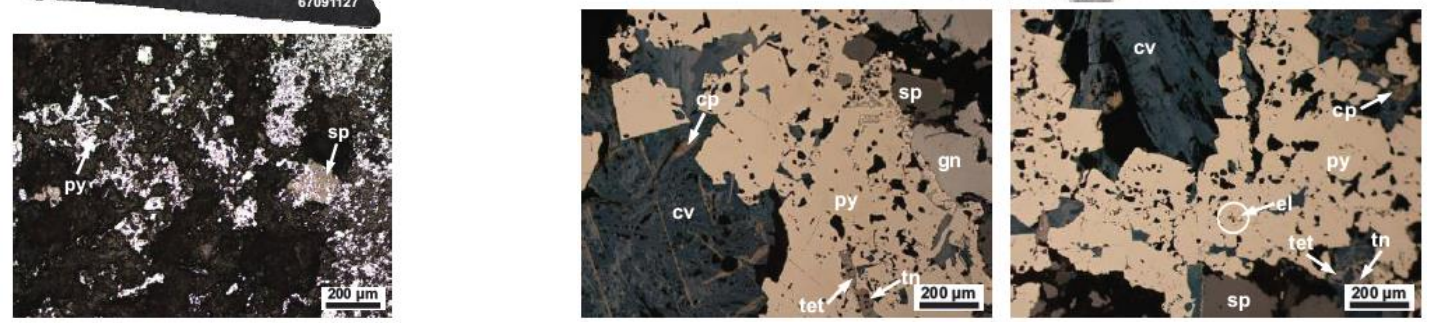

Figure 3. Ore samples with photomicrographs by reflected light showing the association of sulfide minerals for the Toya-Takarada samples of vuggy black-yellow ore, vuggy black ore, quartz-sulfide ore, vuggy black ore (granular pyrite), vuggy black ore (fine-grained pyrite), and black ore. Abbreviations: $c p=$ chalcopyrite, $c v=c o v e l l i t e, ~ e l=e l e c t r u m, g n=g a l e n a$, py=pyrite, $s p=s p h a l e r i t e$, tet=tetrahedrite, $t n=$ tennantite . 
MAKALAH ILMIAH

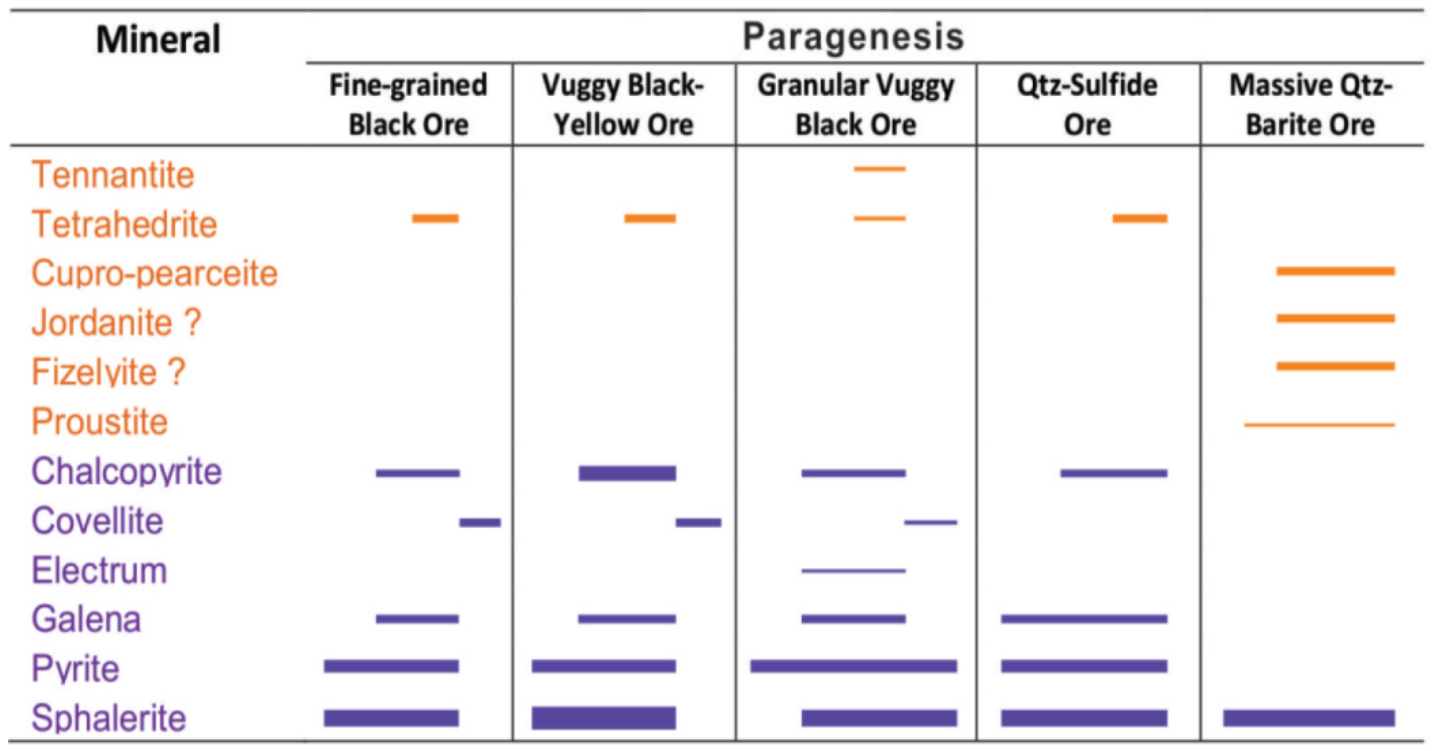

Figure 4. Paragenetic sequence of ore minerals of the Toya-Takarada ores. Width of bars corresponds to the relative abundance of each mineral.

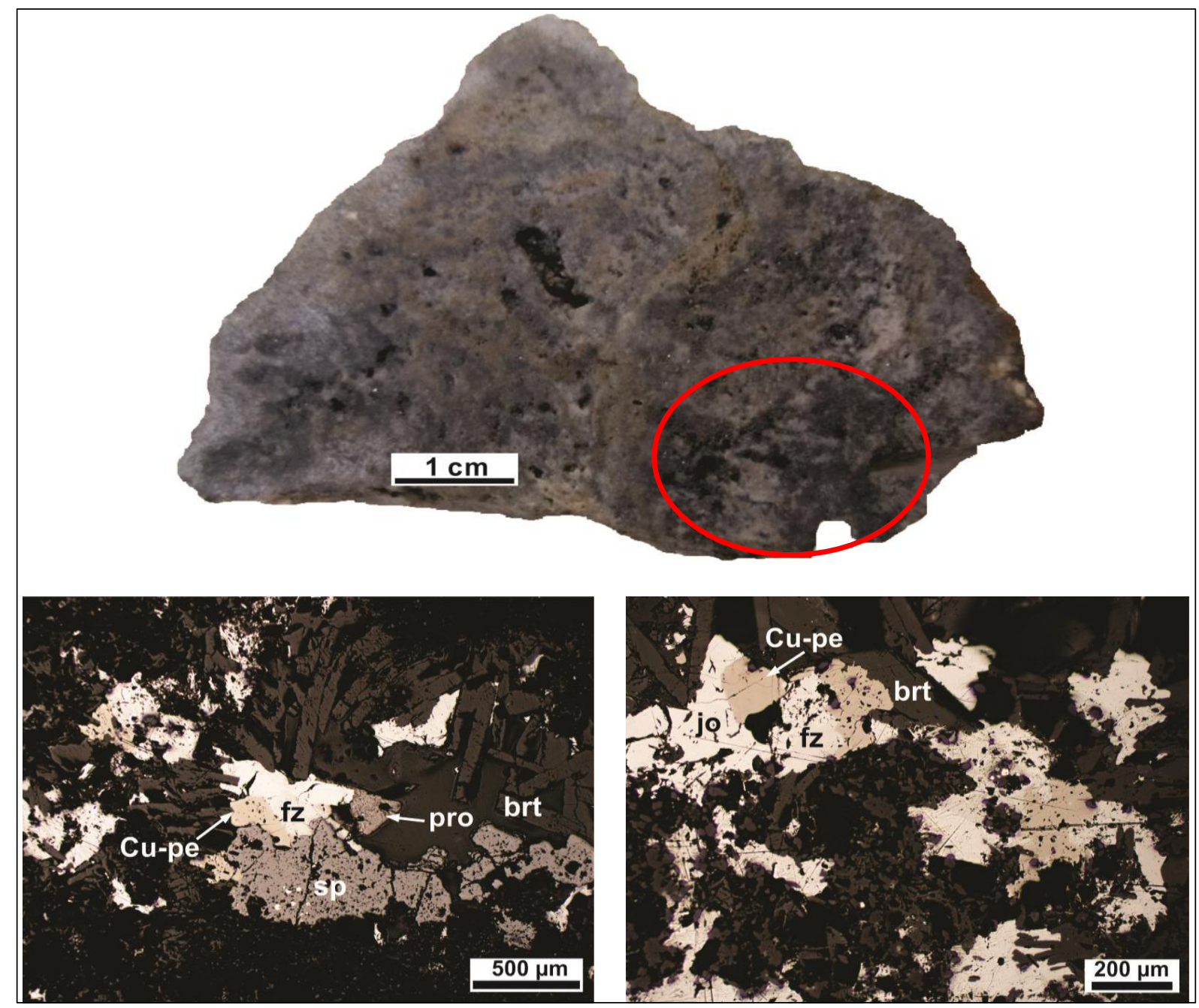

Figure 5. Hand specimen and its photomicrographs of silver sulfosalt minerals in massive quartz-barite ore. Abbreviations: brt=barite, Cu-pe=Cu-rich pearceite, $\mathrm{fz}=\mathrm{fizelyite,}$ jo=jordanite, pro=proustite. 


\section{MAKALAH ILMIAH}
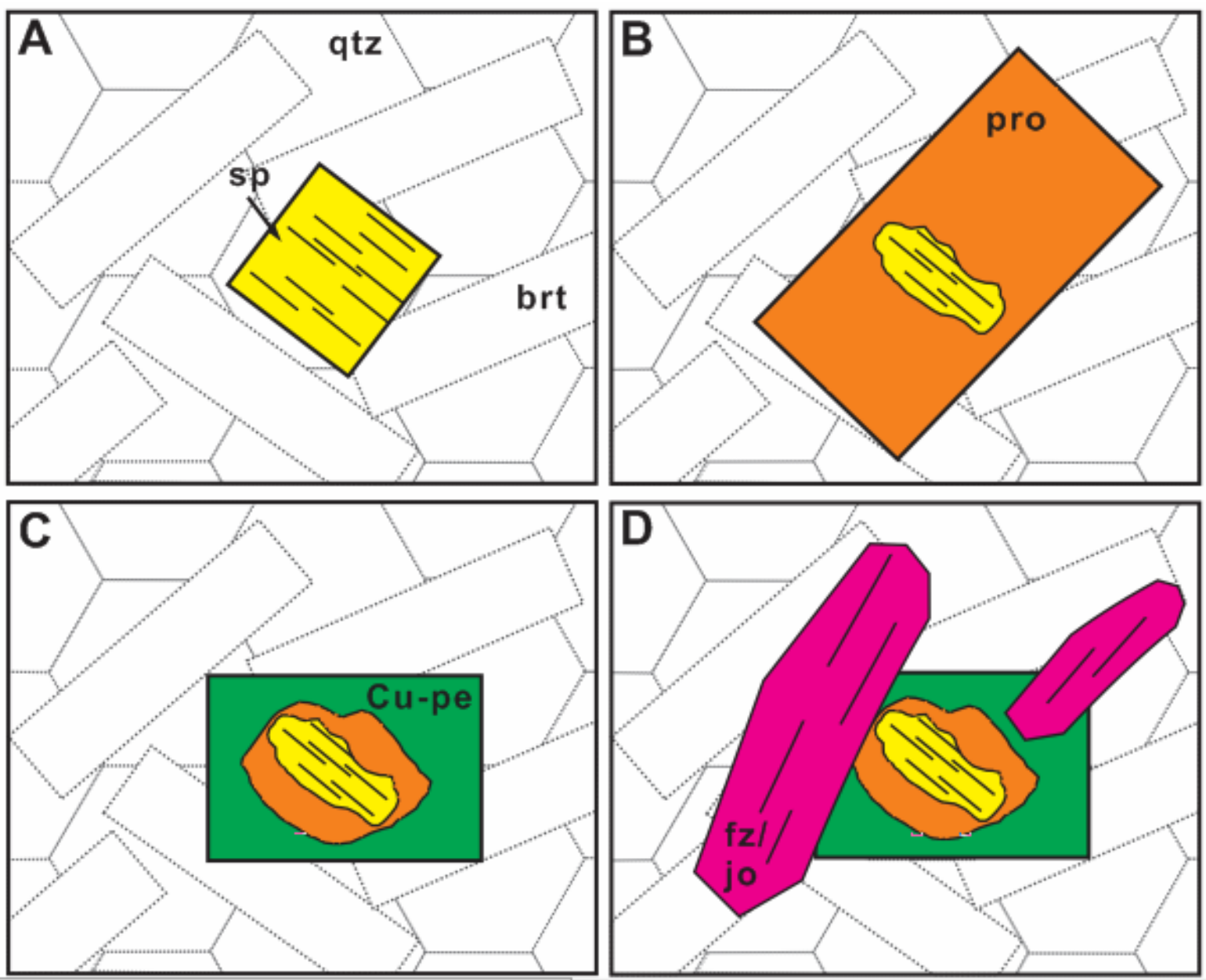

Figure 6. Illustration of the mineralization sequence of the sulfosalt minerals at the ToyaTakarada mine observed in polished sections.

\section{FLUID INCLUSIONS}

A microthermometric study was carried out on the sphalerite samples from the quartzsulfide ore of Toya-Takarada. Prepared quartz samples from this ore were not containing sufficient fluid inclusion to measure further for homogenization temperature or salinity. Otherwise, the primary inclusions observed in sphalerite were large enough to study, aqueous inclusions, mostly consisting of two phases liquid (L) and vapor (V) at room temperature. L-V inclusions are dominant in most inclusions (avg >80 vol\%). However, the vapor bubble occupies up to $\sim 90$ vol\% in some of the inclusions. Although some of the fluid inclusions were necked down, the ones used in the microthermometric study ranged from $10 \mu \mathrm{m}$ to $50 \mu \mathrm{m}$ in diameter
(Figure 7).

The inclusions which occur in cluster or isolated within the crystals is considered as primary and the one in planar arrays outlining growth zones or in healed fractures that terminated against growth zones is considered as pseudo-secondary. The heating and freezing measurements were conducted on fluid inclusions trapped in those minerals using the criteria of Roedder (1984). Within the scope of this study only primary fluid inclusions were measured. The results of measurements from primary fluid inclusions in quartz from different levels indicate that the homogenization temperature is $164-247^{\circ} \mathrm{C}$ (av. $208^{\circ} \mathrm{C}$ ) with salinity ranging from 1.9 to 4.7 wt. $\% \mathrm{NaCl}_{\text {equiv. }}$ (av. 3.9 wt.\% $\mathrm{NaCl}_{\text {equiv. }}$ ) as in Figure 8. 


\section{MAKALAH ILMIAH}

Table 1. Selected results of the electron microprobe analyses for ore minerals from Toya-Takarada Mine.

\begin{tabular}{|c|c|c|c|c|c|c|c|c|c|c|c|c|c|c|}
\hline & & & & & & & Iysis ( & t.\%) & & & & & & \\
\hline & As & Se & Te & $\mathbf{s}$ & $\mathrm{Pb}$ & $\mathrm{Ag}$ & $\mathrm{Cu}$ & $\mathrm{Zn}$ & $\mathrm{Fe}$ & Sb & $\mathrm{Au}$ & $\mathrm{Bi}$ & Total & Calculated formula \\
\hline Tetrahedrite & antite & & & & & & & & & & & & & \\
\hline No.46 & 20.01 & 0.00 & 0.00 & 27.10 & 0.14 & 0.08 & 44.32 & 6.77 & 1.48 & 0.71 & 0.00 & 0.00 & 100.62 & 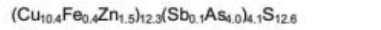 \\
\hline No.47 & 19.47 & 0.00 & 0.00 & 27.33 & 0.08 & 0.05 & 43.47 & 7.29 & 0.74 & 1.80 & 0.25 & 0.00 & 100.47 & $\left(\mathrm{Cu}_{10.2} \mathrm{Fe}_{0.2} \mathrm{Zn}_{1.8}\right)_{12} d\left(\mathrm{Sb}_{0.2} \mathrm{As}_{3.9}\right)_{4,1} \mathrm{~S}_{12.8}$ \\
\hline No.56 & 0.89 & 0.00 & 0.00 & 28.94 & 0.00 & 0.45 & 49.28 & 1.96 & 0.11 & 18.06 & 0.00 & 0.04 & 99.72 & 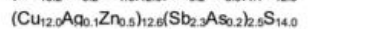 \\
\hline No. 58 & 1.45 & 0.00 & 0.00 & 26.09 & 0.03 & 0.29 & 40.95 & 4.36 & 0.99 & 24.42 & 0.00 & 0.00 & 98.56 & 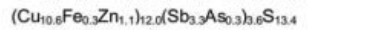 \\
\hline No.59 & 4.68 & 0.00 & 0.00 & 30.40 & 0.22 & 0.06 & 42.04 & 0.62 & 3.73 & 18.22 & 0.67 & 0.00 & 100.65 & $\left(\mathrm{Cu}_{10,1} \mathrm{Fe}_{1.0} \mathrm{Zn}_{0,1}\right)_{1,2}\left(\mathrm{Sb}_{2} \mathrm{As}_{1}, 0 \mathrm{~b}_{3} \mathrm{~S}_{14.5}\right.$ \\
\hline No. 55 & 6.66 & 0.00 & 0.00 & 33.13 & 0.21 & 1.29 & 50.47 & 0.44 & 0.24 & 4.72 & 0.69 & 0.00 & 97.85 & 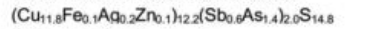 \\
\hline No.122 & 20.96 & 0.00 & 0.00 & 27.25 & 0.00 & 0.06 & 43.87 & 6.72 & 1.73 & 0.00 & 0.00 & 0.00 & 100.59 & 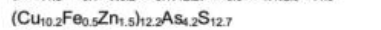 \\
\hline No.124 & 21.02 & 0.00 & 0.08 & 27.28 & 0.00 & 0.03 & 43.63 & 7.31 & 1.10 & 0.08 & 0.19 & 0.00 & 100.72 & $\left(\mathrm{Cu}_{10,2} \mathrm{Fe}_{0,3} \mathrm{Zn}_{1,6}\right)_{12,}, \mathrm{As}_{4,2} \mathrm{~S}_{127}$ \\
\hline No.84 & 2.17 & 0.00 & 0.00 & 24.42 & 0.00 & 4.11 & 36.08 & 7.56 & 0.04 & 24.90 & 0.50 & 0.00 & 99.75 & 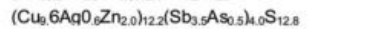 \\
\hline No.89 & 1.74 & 0.00 & 0.00 & 24.45 & 0.10 & 4.54 & 35.31 & 7.78 & 0.08 & 27.02 & 0.20 & 0.00 & 101.22 & 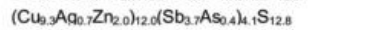 \\
\hline No.83 & 14.06 & 0.00 & 0.00 & 26.63 & 0.20 & 0.85 & 41.04 & 7.92 & 0.41 & 9.04 & 0.25 & 0.00 & 100.38 & 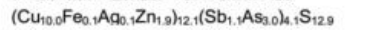 \\
\hline No. 134 & 9.12 & 0.00 & 0.00 & 25.68 & 0.14 & 0.29 & 40.49 & 7.98 & 0.10 & 17.10 & 0.25 & 0.00 & 101.14 & $\left(\mathrm{Cu}_{10,1} \mathrm{Zn}_{1,9}\right)_{120}\left(\mathrm{Sb}_{2,2} \mathrm{As}_{1,9}\right)_{4,1} \mathrm{~S}_{127}$ \\
\hline No.130 & 15.21 & 0.00 & 0.00 & 28.74 & 0.12 & 0.03 & 39.01 & 6.22 & 9.79 & 1.35 & 0.00 & 0.00 & 100.47 & $\left(\mathrm{Cu}_{8,9} \mathrm{Fe}_{2,8} \mathrm{Zn}_{1,4}\right)_{12}\left(\mathrm{Sb}_{0,2} \mathrm{As}_{3,0}\right)_{3,2} \mathrm{~S}_{13,0}$ \\
\hline Mean $(N=13)$ & 10.57 & 0.00 & 0.01 & 27.50 & 0.10 & 0.93 & 42.30 & 5.61 & 1.58 & 11.34 & 0.23 & 0.00 & 100.16 & $\left(\mathrm{Cu}_{10,3} \mathrm{Fe}_{0,4} \mathrm{Ag}_{0,1}, \mathrm{Zn}_{1,3}\right)_{12,1}\left(\mathrm{Sb}_{1,3} \mathrm{As}_{2,1}\right)_{2,0} \mathrm{~S}_{13,2}$ \\
\hline Proustite & & & & & & & & & & & & & & \\
\hline No.92 & 10.24 & 0.00 & 0.16 & 19.21 & 0.00 & 67.99 & 1.12 & 0.04 & 0.04 & 0.13 & 0.11 & 0.00 & 99.03 & $\mathrm{Aa}_{3.3}\left(\mathrm{As}_{0,7} \mathrm{Cu}_{0,3}\right)_{6.8} \mathrm{~S}_{3.0}$ \\
\hline No.14 & 12.13 & 0.00 & 0.11 & 19.37 & 0.00 & 67.94 & 1.17 & 0.00 & 0.00 & 0.01 & 0.00 & 0.00 & 100.74 & $\mathrm{Aq}_{3.2}\left(\mathrm{As}_{0,7} \mathrm{Cu}_{0,7}\right) \mathrm{S}_{0.8}$ \\
\hline No. 15 & 11.96 & 0.00 & 0.40 & 19.10 & 0.00 & 66.92 & 1.16 & 0.04 & 0.00 & 0.00 & 0.17 & 0.00 & 99.74 & $\mathrm{Ag}_{3.2 .}\left(\mathrm{As}_{0.7} \mathrm{Cu}_{0.10 .8}\right)_{0.8} \mathrm{~S}_{3.0}$ \\
\hline Mean $(N=3)$ & 11.44 & 0.00 & 0.22 & 19.23 & 0.00 & 67.62 & 1.15 & 0.03 & 0.01 & 0.05 & 0.09 & 0.00 & 99.84 & $A q_{3,3}\left(\mathrm{As}_{0.7} \mathrm{Cu}_{0,1}\right)_{0.3} \mathbf{S}_{3.0}$ \\
\hline Cupropearc & & & & & & & & & & & & & & \\
\hline No.93 & 15.10 & 0.00 & 0.00 & 28.29 & 0.07 & 25.78 & 29.21 & 0.00 & 0.00 & 1.91 & 0.32 & 0.00 & 100.68 & $\mathrm{Ag}_{3.9} \mathrm{Cu}_{7}\left(\mathrm{As}_{3} \mathrm{SB}_{0,3} \mathrm{~S}_{6} \mathrm{~S}_{142}\right.$ \\
\hline No.99 & 15.94 & 0.00 & 0.00 & 29.03 & 0.00 & 24.16 & 29.64 & 0.00 & 0.00 & 2.42 & 0.09 & 0.00 & 101.29 & $\mathrm{Ag}_{3 . .6} \mathrm{Cu}_{\mathrm{H}_{A} A}\left(\mathrm{As}_{3,4} \mathrm{Sb}_{0,3}\right)_{27} \mathrm{~S}_{14,4}$ \\
\hline No.101 & 16.64 & 0.00 & 0.00 & 28.66 & 0.00 & 24.56 & 29.40 & 0.00 & 0.01 & 1.63 & 0.15 & 0.00 & 101.04 & $\mathrm{Aq}_{9_{6} .6} \mathrm{Cu}_{7}\left(\mathrm{As}_{3.5} \mathrm{Sb}_{0.2}\right)_{77} \mathrm{~S}_{142}$ \\
\hline No.13 & 15.18 & 0.00 & 0.00 & 28.37 & 0.11 & 26.39 & 28.77 & 0.04 & 0.00 & 1.40 & 0.90 & 0.00 & 101.15 & $\mathrm{Ad}_{29} \mathrm{Cu}_{7}\left(\mathrm{As}_{3}, 3 \mathrm{Sb}_{02}\right)_{35} \mathrm{~S}_{14.4}$ \\
\hline No.16 & 16.33 & 0.00 & 0.00 & 29.05 & 0.00 & 24.09 & 29.25 & 0.03 & 0.00 & 1.35 & 0.00 & 0.00 & 100.11 & $\mathrm{Ad}_{3.6} \mathrm{Cu}_{7,3}\left(\mathrm{As}_{3,5} \mathrm{Sb}_{02} \mathrm{~h}_{7} \mathrm{~S}_{14.4}\right.$ \\
\hline No.23 & 16.12 & 0.00 & 0.00 & 29.07 & 0.00 & 23.60 & 29.76 & 0.00 & 0.00 & 0.95 & 0.00 & 0.00 & 99.50 & $\mathrm{Ag}_{9.5} \mathrm{Cu}_{\mathrm{H}_{5} s}\left(\mathrm{As}_{3,4} \mathrm{Sb}_{0,1}\right)_{5,5} \mathrm{~S}_{145}$ \\
\hline Mean $(N=6)$ & 15.89 & 0.00 & 0.00 & 28.75 & 0.03 & 24.76 & 29.34 & 0.01 & 0.00 & 1.61 & 0.24 & 0.00 & 100.63 & 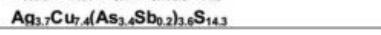 \\
\hline Fizelyite & & & & & & & & & & & & & & \\
\hline No.94 & 1.47 & 0.00 & 0.00 & 21.46 & 35.27 & 5.56 & 0.06 & 0.00 & 0.00 & 32.63 & 0.00 & 0.00 & 96.44 & $\mathrm{Ad}_{3,9}\left(\mathrm{~Pb}_{12,8} \mathrm{Cu}_{0,1}\right)_{129} \mathrm{Sb}_{19,9} \mathrm{~S}_{50,3}$ \\
\hline No.95 & 1.31 & 0.00 & 0.00 & 21.65 & 37.00 & 5.78 & 0.04 & 0.00 & 0.00 & 32.98 & 0.17 & 0.00 & 98.92 & 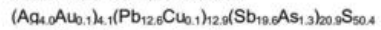 \\
\hline No.96 & 1.61 & 0.00 & 0.00 & 21.55 & 36.43 & 5.95 & 0.00 & 0.00 & 0.02 & 32.84 & 0.02 & 0.00 & 98.42 & $\mathrm{Aq}_{1,1} \mathrm{~Pb}_{12} \pi\left(\mathrm{Sb}_{19,5} \mathrm{As}_{1.8}\right)_{21,1} \mathrm{~S}_{50,1}$ \\
\hline No.97 & 1.31 & 0.00 & 0.00 & 21.51 & 36.23 & 6.49 & 0.00 & 0.01 & 0.00 & 33.71 & 0.00 & 0.00 & 99.25 & 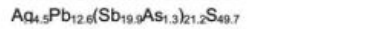 \\
\hline No. 100 & 1.17 & 0.00 & 0.00 & 21.88 & 36.46 & 6.47 & 0.00 & 0.00 & 0.02 & 33.79 & 0.00 & 0.00 & 99.79 & 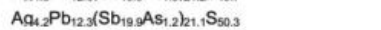 \\
\hline No. 12 & 0.69 & 0.00 & 0.00 & 21.40 & 36.40 & 6.34 & 0.01 & 0.00 & 0.00 & 33.32 & 0.00 & 0.00 & 98.16 & $\mathrm{Aq}_{1.5} \mathrm{~Pb}_{12.0}\left(\mathrm{Sb}_{20.0} \mathrm{As}_{0,7}\right)_{20,7} \mathrm{~S}_{49.9}$ \\
\hline No.19 & 0.98 & 0.00 & 0.00 & 21.80 & 36.46 & 6.97 & 0.02 & 0.00 & 0.02 & 32.59 & 0.59 & 0.00 & 99.43 & 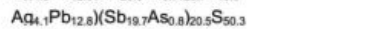 \\
\hline No.21 & 0.63 & 0.00 & 0.00 & 21.49 & 36.43 & 6.38 & 0.02 & 0.00 & 0.03 & 33.25 & 0.00 & 0.00 & 98.22 & $\mathrm{Aq}_{4.4} \mathrm{~Pb}_{12}, \mathrm{Sb}_{20.3} \mathrm{As}_{0,8} h_{20.9} \mathrm{~S}_{49.9}$ \\
\hline No.22 & 1.07 & 0.00 & 0.00 & 21.58 & 35.33 & 6.70 & 0.07 & 0.01 & 0.00 & 33.17 & 0.08 & 0.00 & 98.01 & 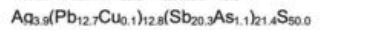 \\
\hline Mean $(N=9)$ & 1.14 & 0.00 & 0.00 & 21.59 & 36.22 & 6.29 & 0.02 & 0.00 & 0.01 & 33.14 & 0.10 & 0.00 & 98.52 & 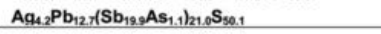 \\
\hline Jordanite & & & & & & & & & & & & & & \\
\hline No.102 & 2.49 & 0.00 & 0.00 & 17.22 & 67.50 & 0.18 & 0.00 & 0.03 & 0.00 & 11.57 & 0.05 & 0.00 & 99.03 & $\left(\mathrm{~Pb}_{13,3} \mathrm{Aq}_{0.1}\right)_{14.0}\left(\mathrm{As}_{1.5} \mathrm{Sb}_{4,3}\right)_{5.8} \mathrm{~S}_{232}$ \\
\hline No.17 & 2.15 & 0.00 & 0.00 & 16.87 & 68.07 & 0.01 & 0.05 & 0.00 & 0.00 & 11.59 & 0.32 & 0.00 & 99.06 & $\left(\mathrm{~Pb}_{14,} \mathrm{AL}_{0,1}\right)_{1,5}\left(\mathrm{As}_{1,3} \mathrm{Sb}_{4,2}\right)_{5.5} \mathrm{~S}_{23.0}$ \\
\hline No. 18 & 2.44 & 0.00 & 0.00 & 17.03 & 67.88 & 0.41 & 0.00 & 0.01 & 0.04 & 12.24 & 0.32 & 0.00 & 100.37 & $\left(\mathrm{~Pb}_{14,1} \mathrm{Ag}_{0,2} \mathrm{Au}_{0.1}\right)_{1,4.4}\left(\mathrm{As}_{1,4} \mathrm{Sb}_{4,3}\right)_{5,7} \mathrm{~S}_{22.9}$ \\
\hline No.20 & 2.51 & 0.00 & 0.00 & 17.41 & 67.22 & 0.23 & 0.20 & 0.00 & 0.00 & 11.54 & 0.00 & 0.00 & 99.12 & $\left(\mathrm{~Pb}_{14,0} \mathrm{Cu}_{0,1} \mathrm{Ag}_{0,1}\right)_{1,2}\left(\mathrm{As}_{1.5} \mathrm{Sb}_{4,1}\right)_{2.6} \mathrm{~S}_{232}$ \\
\hline Mean $(N=4)$ & 2.40 & 0.00 & 0.00 & 17.13 & 67.67 & 0.21 & 0.06 & 0.01 & 0.01 & 11.74 & 0.17 & 0.00 & 99.40 & $\left(\mathbf{P b}_{14}, \mathbf{A q}_{0,1}\right)_{42}\left(\mathbf{A s}_{1,1} \mathbf{S b}_{42,}\right)_{6,6} \mathbf{S}_{23,1}$ \\
\hline Sphalerite & & & & & & & & & & & & & & \\
\hline No.44 & 0.00 & 0.00 & 0.00 & 32.19 & 0.11 & 0.07 & 0.08 & 65.72 & 0.59 & 0.07 & 0.39 & 0.11 & 99.33 & $\mathrm{Zm}_{1.0} \mathrm{~s}_{1.0}$ \\
\hline No.48 & 0.08 & 0.00 & 0.00 & 32.24 & 0.08 & 0.00 & 0.05 & 66.32 & 0.32 & 0.00 & 0.32 & 0.00 & 99.41 & $\mathrm{Zn}_{1,0} \mathrm{~S}_{1.0}$ \\
\hline No.54 & 0.00 & 0.00 & 0.03 & 32.90 & 0.00 & 0.07 & 0.04 & 65.57 & 0.57 & 0.13 & 0.63 & 0.00 & 99.93 & $\mathrm{Zn}_{1.0} \mathrm{~S}_{1.0}$ \\
\hline No.61 & 0.00 & 0.00 & 0.00 & 32.48 & 0.05 & 0.00 & 0.12 & 65.15 & 0.45 & 0.11 & 0.22 & 0.00 & 98.57 & $\mathrm{Zn}_{1.0 \mathrm{~S}_{1.0}}$ \\
\hline No. 123 & 0.00 & 0.00 & 0.02 & 31.65 & 0.27 & 0.00 & 0.00 & 66.03 & 0.37 & 0.03 & 0.04 & 0.12 & 98.53 & $\mathrm{Zn}_{1.0} \mathrm{~S}_{1.0}$ \\
\hline No. 85 & 0.00 & 0.00 & 0.06 & 32.77 & 0.00 & 0.00 & 0.04 & 65.43 & 0.65 & 0.00 & 0.28 & 0.17 & 99.40 & $\mathrm{Zn}_{1,0} \mathrm{~S}_{1,0}$ \\
\hline No.90 & 0.00 & 0.00 & 0.11 & 32.72 & 0.00 & 0.04 & 0.06 & 65.91 & 0.58 & 0.11 & 0.82 & 0.00 & 100.34 & $\mathrm{Zn}_{\mathrm{n} . \mathrm{S}_{1.0}}$ \\
\hline No.98 & 0.00 & 0.00 & 0.02 & 32.13 & 0.11 & 0.14 & 0.08 & 65.28 & 0.15 & 0.50 & 0.00 & 0.02 & 98.42 & $\mathrm{Zn}_{1,0} \mathrm{~s}_{1,0}$ \\
\hline No. 131 & 0.00 & 0.00 & 0.01 & 32.73 & 0.02 & 0.04 & 0.05 & 66.10 & 0.72 & 0.03 & 1.22 & 0.02 & 100.93 & $\mathrm{Zn}_{1.0} \mathrm{~S}_{1.0}$ \\
\hline No.135 & 0.00 & 0.00 & 0.00 & 32.56 & 0.00 & 0.02 & 0.06 & 65.74 & 0.66 & 0.02 & 0.78 & 0.32 & 100.16 & $\mathrm{Zn}_{1.0 \mathrm{~S}_{1.0}}$ \\
\hline Mean $(N=10)$ & 0.01 & 0.00 & 0.02 & 32.44 & 0.06 & 0.04 & 0.06 & 65.72 & 0.51 & 0.10 & 0.47 & 0.08 & 99.50 & $\mathrm{Zn}_{1,0} \mathrm{~s}_{1,0}$ \\
\hline Chalcopyrit & & & & & & & & & & & & & & \\
\hline No.43 & 0.00 & 0.00 & 0.05 & 33.14 & 5.05 & 0.04 & 32.63 & 0.08 & 29.39 & 0.00 & 0.00 & 0.00 & 100.37 & $\mathrm{Cu}_{1 .}, \mathrm{Fe}_{1.0 \mathrm{~S}_{2.0}}$ \\
\hline No.45 & 0.00 & 0.00 & 0.06 & 34.34 & 0.06 & 0.24 & 34.53 & 0.38 & 30.27 & 0.00 & 0.66 & 0.08 & 100.61 & $\mathrm{Cu}_{1 .} \mathrm{Fe}_{1.0 \mathrm{~S}_{20}}$ \\
\hline No. 57 & 0.00 & 0.00 & 0.00 & 31.28 & 0.00 & 1.42 & 50.51 & 0.00 & 14.59 & 0.00 & 0.67 & 0.00 & 98.47 & $\mathrm{Cu}_{1.6} \mathrm{Fe}_{0.5} \mathrm{~S}_{1.9}$ \\
\hline Mean $(N=3)$ & 0.00 & 0.00 & 0.03 & 32.92 & 1.70 & 0.57 & 39.22 & 0.15 & 24.75 & 0.00 & 0.45 & 0.03 & 99.81 & $\mathrm{Cu}_{1,2} \mathrm{Fe}_{0,0} \mathrm{~S}_{20}$ \\
\hline Pvrite & & & & & & & & & & & & & & \\
\hline No. 51 & 0.00 & 0.00 & 0.00 & 53.50 & 0.00 & 0.00 & 0.10 & 0.02 & 47.57 & 0.00 & 0.33 & 0.26 & 101.79 & $\mathrm{Fe}_{1,0} \mathrm{~S}_{2,0}$ \\
\hline Mean $(N=1)$ & 0.00 & 0.00 & 0.00 & 53.50 & 0.00 & 0.00 & 0.10 & 0.02 & 47.57 & 0.00 & 0.33 & 0.26 & 101.79 & $\mathrm{Fe}_{1.0} \mathrm{~S}_{20}$ \\
\hline Galena & & & & & & & & & & & & & & \\
\hline No. 60 & 0.00 & 0.00 & 0.05 & 13.28 & 87.62 & 0.00 & 0.01 & 0.00 & 0.05 & 0.01 & 0.24 & 0.00 & 101.26 & $\mathrm{~Pb}_{1.0 \mathrm{~S}} \mathrm{~S}_{1,0}$ \\
\hline No. 127 & 0.00 & 0.00 & 0.00 & 13.13 & 85.75 & 0.00 & 0.56 & 1.08 & 0.52 & 0.00 & 0.00 & 0.00 & 101.03 & $\mathrm{~Pb}_{1,0} \mathrm{~S}_{1,0}$ \\
\hline No.87 & 0.00 & 0.00 & 0.00 & 13.09 & 87.39 & 0.00 & 0.14 & 0.03 & 0.00 & 0.04 & 0.00 & 0.00 & 100.68 & $\mathrm{~Pb}_{1.0} \mathrm{~S}_{1.0}$ \\
\hline No.129 & 0.00 & 0.00 & 0.10 & 13.08 & 87.73 & 0.00 & 0.11 & 0.03 & 0.06 & 0.09 & 0.00 & 0.00 & 101.20 & $\mathrm{~Pb}_{1.0} \mathrm{~S}_{1.0}$ \\
\hline No.136 & 0.00 & 0.00 & 0.12 & 13.39 & 87.38 & 0.00 & 0.25 & 0.42 & 0.00 & 0.26 & 0.28 & 0.00 & 102.09 & $\mathrm{~Pb}_{1.0} \mathrm{~S}_{1.0}$ \\
\hline Mean $(N=5)$ & 0.00 & 0.00 & 0.05 & 13.19 & 87.17 & 0.00 & 0.21 & 0.31 & 0.12 & 0.08 & 0.10 & 0.00 & 101.25 & $\mathrm{~Pb}_{1.0} \mathrm{~S}_{1.0}$ \\
\hline Covellite & & & & & & & & & & & & & & \\
\hline No.49 & 0.09 & 0.00 & 0.00 & 32.48 & 0.00 & 0.02 & 68.27 & 0.00 & 0.03 & 0.00 & 0.00 & 0.00 & 100.90 & $\mathrm{Cu}_{1.0} \mathrm{~S}_{10}$ \\
\hline No.91 & 0.00 & 0.00 & 0.08 & 30.16 & 0.32 & 8.26 & 61.30 & 0.14 & 0.06 & 0.00 & 0.32 & 0.00 & 100.65 & $\mathrm{Cu}_{1.0 \mathrm{~S}} \mathrm{~S}_{1.0}$ \\
\hline Mean $(N=2)$ & 0.04 & 0.00 & 0.04 & 31.32 & 0.16 & 4.14 & 64.78 & 0.07 & 0.05 & 0.00 & 0.16 & 0.00 & 100.78 & $\mathrm{Cu}_{1.0} \mathrm{~S}_{1,0}$ \\
\hline Electrum & & & & & & & & & & & & & & \\
\hline No. 50 & 0.00 & 0.00 & 0.00 & 0.96 & 0.00 & 20.68 & 0.25 & 0.02 & 0.46 & 0.00 & 77.29 & 0.00 & 99.66 & $A u_{1}, 3 g_{0.7}$ \\
\hline No.52 & 0.00 & 0.00 & 0.01 & 0.81 & 0.00 & 19.38 & 0.30 & 0.00 & 0.97 & 0.00 & 76.81 & 0.00 & 98.28 & $A U_{1} \cdot A_{g_{2}}$ \\
\hline No. 53 & 0.00 & 0.00 & 0.04 & 0.73 & 0.00 & 20.35 & 1.56 & 0.06 & 0.22 & 0.13 & 76.25 & 0.00 & 99.33 & $\mathrm{Au}_{1.3} \mathrm{~A} \mathrm{~g}_{0.7}$ \\
\hline Mean $(N=3)$ & 0.00 & 0.00 & 0.01 & 0.83 & 0.00 & 20.14 & 0.70 & 0.03 & 0.55 & 0.04 & 76.78 & 0.00 & 99.09 & $\mathbf{A} \mathbf{u}_{1,3} \mathbf{A} \mathbf{A g}_{0.7}$ \\
\hline
\end{tabular}




\section{MAKALAH ILMIAH}

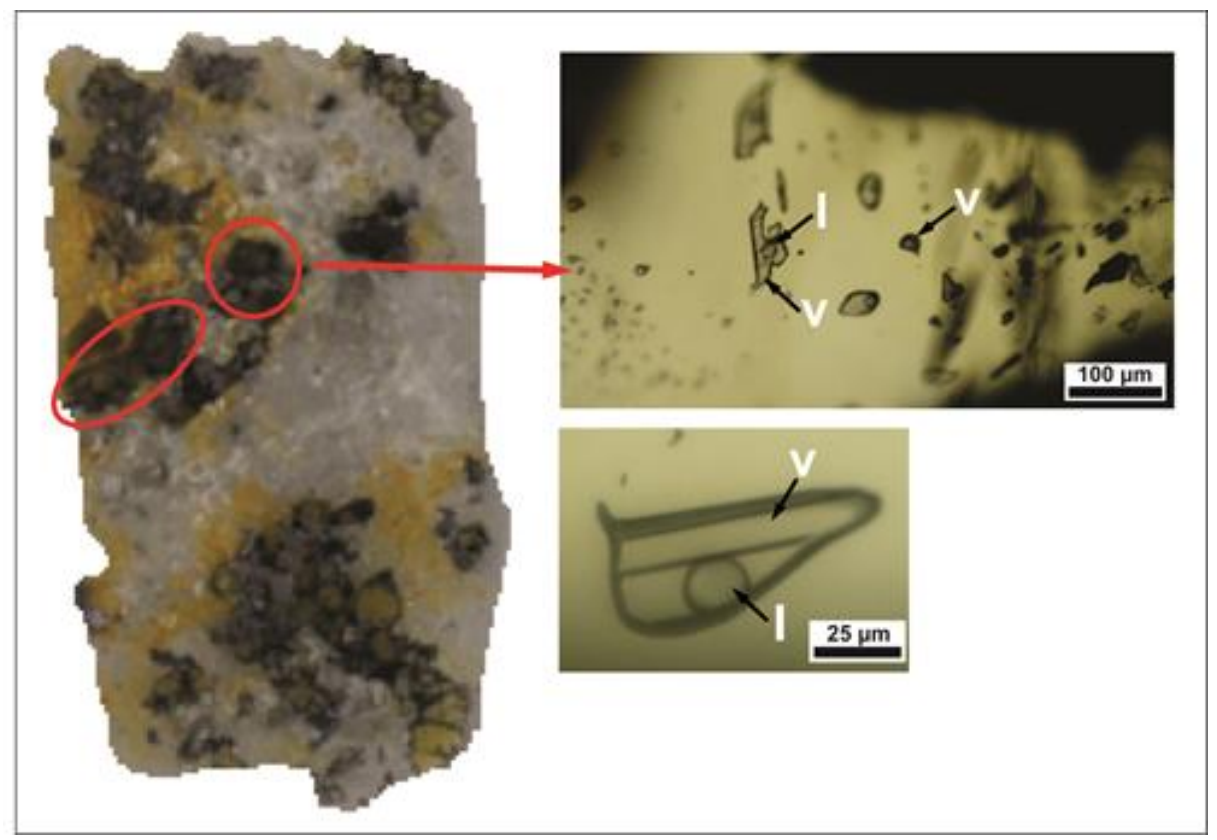

Figure 7. Photomicrographs by transmitted-light of fluid inclusions trapped in sphalerite and quartz of quartz-sulfide ore.

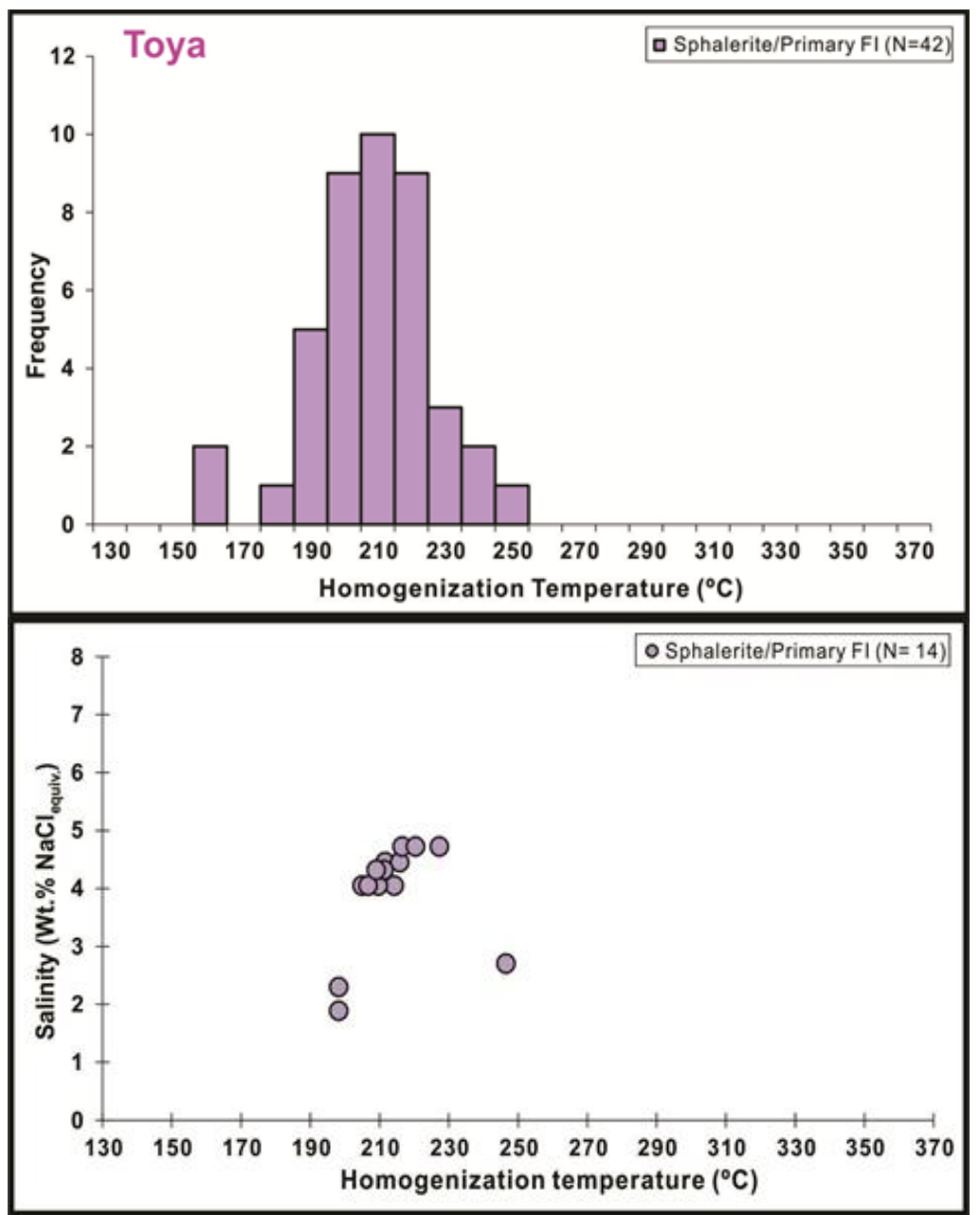

Figure 8. Ranges of homogenization temperature (upper) and salinity (lower) measured for fluid inclusion in sphalerite mineral. 
Earlier studies on fluid inclusions in Kuroko samples (Pisutha-Arnond and Ohmoto, 1983) shows two important characteristics of the ore forming fluids such as the temperatures were around $250^{\circ} \mathrm{C}$, and the salinities were similar to those of normal seawater (i.e., $\sim 3.5$ wt. \% $\mathrm{NaCl}_{\text {equiv. }}$ ). such fluids may rise like a plume when discharged into the overlying sea; their densities at $\mathrm{T} \sim 250^{\circ} \mathrm{C}$ are only about 0.7 . Mixing of the plume fluids with cold seawater may cause ore minerals to crystallize out in the plume and settle on the seafloor.

The hydrothermal fluids chemical conditions during ore and gangue minerals deposition in the Toya-Takarada is estimated by geochemical equilibrium thermodynamics of ore deposition. The phase relations together with mineral compositions in the systems Fe-Zn-S (Scott and Barnes, 1971) were used to estimate the ranges of temperature and sulfur fugacity $\left(f S_{2}\right)$ for gold-silver-base metal mineralization of Toya-Takarada.. The sulfur fugacity and temperature conditions for the ore mineralization at the Toya-Takarada is shown in Figure 9.

The precipitation of the base metals- and precious metals-bearing minerals were occurred at the high sulfur fugacity by an additional rising of the hydrothermal fluid and the opening of the fractures. The close relationship of gold with sulfide and qurtzsulfide ores suggests that the gold and the base metals were transported and deposited by similar fluids. It is suggested that the solution with sulfur fugacity around $10^{-16}$ to $10^{-10}$ is the main mechanism for the deposition of gold and base metals in the Toya Takarada.

Kuroko-type deposits in Japan including the Toya-Takarada mine have the major constituents of the deposits of pyrite, chalcopyrite, sphalerite, galena, barite and quartz. They are zoned vertically with gypsum and/or anhydrite commonly occurs as separate bodies beside the sulphidebarite bodies.
The zoning of minerals may have resulted from changes in density of the ore solutionsea water mixtures as precipitation proceeded. In Japan, it is thought that the ores were precipitated on the Miocene seafloor from saline solutions in a weakly acidic, reducing environment at about $200-250^{\circ} \mathrm{C}$ as a result of falling temperatures (Sato, 1977).

Previous studies of light stable isotopes suggest that the mineralizing solutions were predominantly coeval sea water. Lead isotope data, together with base-metal contents of the host rocks, however, indicate that leaching by circulating sea water cannot have been the dominant mechanism. So, magmatic water probably played an important role in the mineralization. According to Sato (1977), about 20-25 Ma, as the Pacific plate was being subducted, a large amount of basic to intermediate magma was generated and differentiated in the lower crustal region. Magmatic fluids produced at the last stage of differentiation, about $13 \mathrm{Ma}$, rose to the sea-floor and, on mixing with sea water, produced the Kuroko deposits. Subsidence of the sedimentary basins before the felsic volcanism could have been the result of volume decrease during solidification of underlying magma.

Otherwise, Ohmoto (1996) suggests mixing of the ore-forming fluids with local seawater within unconsolidated sediments and/or on the seafloor causes precipitation of "primitive ores" with the black ore mineralogy (sphalerite + galena + pyrite + barite + anhydrite). Then, the reactions between the "primitive ores" with later and hotter hydrothermal fluids cause transformation of "primitive ores" to "matured ores" that are enriched in chalcopyrite and pyrite.

In the condition of hydrothermal fluids contained sufficient amounts of both $\mathrm{H}_{2} \mathrm{~S}$ and the heavy metals, mixing of hydrothermal fluid with cold seawater is a very effective mechanism for precipitation of sphalerite and galena (and tetrahedritetennantite) that form black ores. The 


\section{MAKALAH ILMIAH}

saturation indices for these minerals increase continuously with increasing proportions of cold seawater in the mixtures. During mixing of hot hydrothermal fluid and cold seawater, the common Fe-bearing minerals of pyrite (in some other Kuroko deposits is hematite, goethite, pyrrhotite and less common magnetite) may precipitate.

However Urabe and Marumo (1991) noted the lead isotopic study and $\mathrm{Pb} / \mathrm{Zn}$ ratios in deposits of the northeastern Pacific Ocean indicate that some lead, as well as other metals such as barium and zinc, came from the wall rock through a leaching process.

\section{CONCLUSIONS}

In general, the ore minerals in Kuroko-type deposits of Toya-Takarada occurred mainly in the black and yellow ores zone dominantly composed of sphalerite, galena, chalcopyrite and pyrite in association with tetrahedrite-tennantite series. The massive quartz-barite ore sample of Toya-Takarada mine are also contain some rare silver sulfosalt minerals such as proustite, Curich pearceite, geocronite-jordanite and fizelyite. Those minerals were found together in association with sphalerite. The ore minerals composition of common sulfides has the ideal IMA-approved formula. The sulfides ores are zoned vertically with gypsum and/or anhydrite commonly occurs as separate bodies beside the sulphide-barite bodies.

The formation temperature and salinity measured from sphalerite are in the averages of $208^{\circ} \mathrm{C}$ and $3.9 \mathrm{wt} . \% \mathrm{NaCl}_{\text {equiv. }}$.

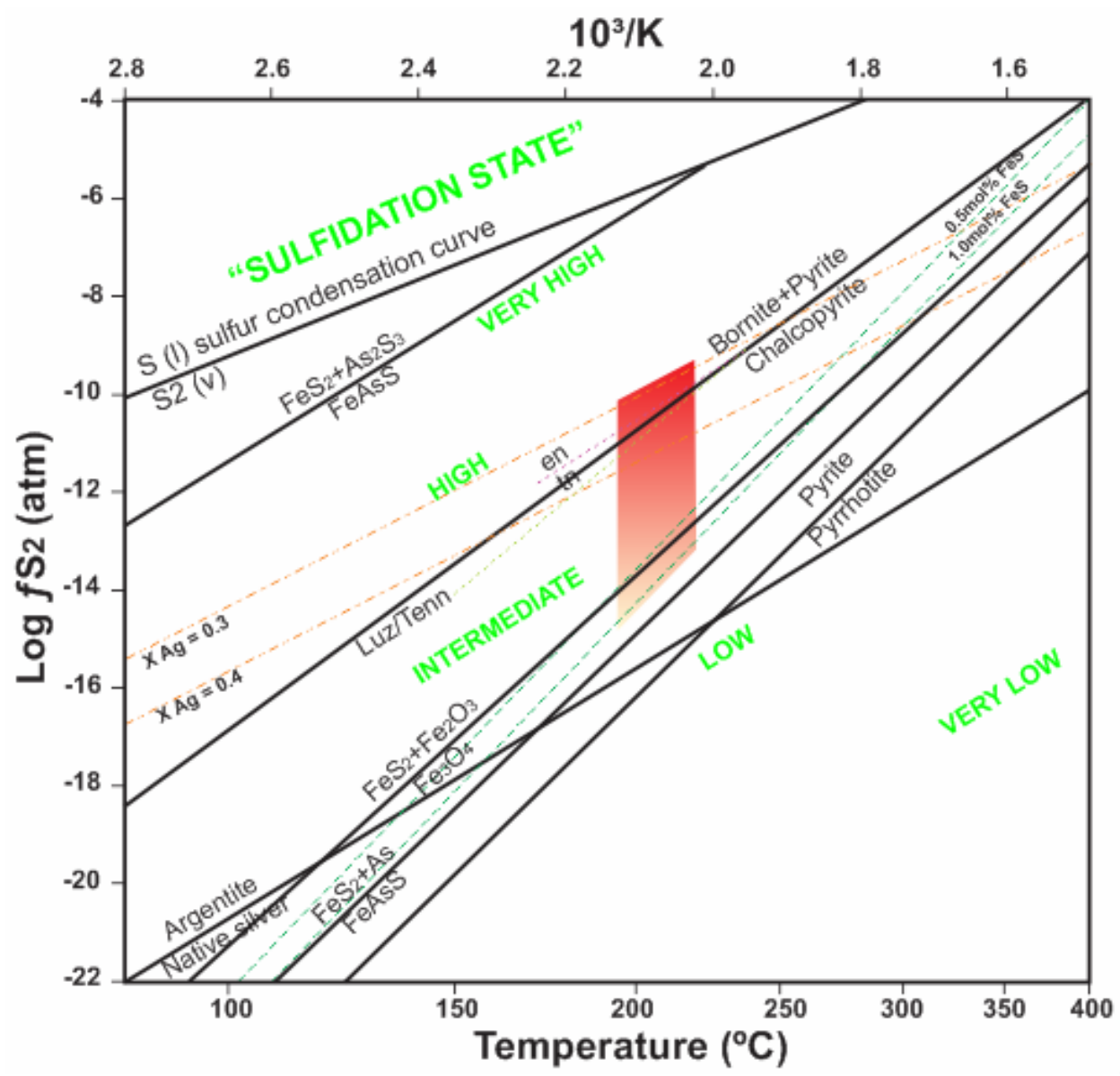

Figure 9. $\log f S_{2}$ versus temperature conditions for the mineralization at the Toya-Takarada deposit. Isopleths of FeS content for sphalerite are calculated using equations of Scott \&

Barnes (1971). The shaded areas approximate the sulfur fugacity and temperature conditions for mineralization 
The mineral assemblage, iron content in sphalerite and silver content in electrum indicated that sulfur fugacity was slightly high during ore mineralization in ToyaTakarada mine. The stable isotope study of sulfur, oxygen and hydrogen is required to identify the source of mineralizing solutions and to prove the possibility that magmatic water played an important role in the mineralization comparing to the leaching by circulating sea water.

\section{ACKNOWLEDGEMENT}

This research project was financially supported by Ministry of Research, Technology and Higher Education of the Republic of Indonesia and Postdoctoral Fellowship Program of Japan Society for Promotion of Science (JSPS).

\section{REFERENCES}

Marumo, K., 1985. Geological and mineralogical studies on alteration zones of Lake Toya area, Southwestern Hokkaido. Mining Geologi, 35, 5, p.331-344, in Japanese with English abstract.

Marumo, K. and Sawai, O., 1986. K-Ar ages of some vein-type and Kurokotype deposits in southwestern Hokkaido, Japan. Mining Geologi, 36, 1, p.21-26, in Japanese with English abstract.

Roedder, E., 1984. Fluid Inclusions. Reviews in Mineralogy, 12, Mineralogical Society of America, Washington D. C, 644p.
Ohmoto, H., 1996. Formation of volcanogenic massive sulfide deposits: The Kuroko perspective. Ore Geology Reviews, 10, p.135-177.

Pisutha-Arnond, V. and Ohmoto, H., 1983. Thermal history, and chemical and isotopic compositions of the oreforming fluids responsible for the Kuroko massive sulfide deposits in the Hokuroku district of Japan. International Journal of Economic Geology, Monograf, 5, p523-558.

Sato, T., 1977. Kuroko deposits: their geology, geochemistry and origin. Journal of Geological Society, Special Publications, 7, p153-161.

Sawai, O. and Itaya, T., 1993. K-Ar ages of Kuroko-type deposits in the Shakotan-Toya district, Southwest Hokkaido, Japan. International Journal of Resource Geology, 43, 3, p.165-172.

Scott, S. D. and Barnes, H. L., 1971. Sphalerite geothermometry and geobarometry. International Journal of Economic Geology, 66, p653-669.

Shikazono, N. and Shimizu, M., 1988. Electrum: chemical composition, mode of occurrence and depositional environment. University of Museum, University Tokyo Bulletin, 32, 81p.

Urabe, T. and Marumo, K., 1991. A new model for Kuroko-type deposits of Japan, Episodes, 14, 3, p246-251.

Yuningsih, E. T. and Matsueda, H., 2013. Silver sulfosalt minerals from the Toya-Takarada mine, Southwest Hokkaido, Japan. 63 ${ }^{\text {rd }}$ Annual Meeting of the Society of Resource Geology (abstract vol.), P-04, p.64.

\begin{tabular}{|ll|}
\hline Diterima & $:$ 23 Juni 2016 \\
Direvisi & $:$ 28 Juli 2016 \\
Disetujui & $:$ 19 Agustus 2016 \\
\hline
\end{tabular}

